\title{
A NOTE ON THE WELL-POSEDNESS \\ OF TERMINAL VALUE PROBLEMS FOR FRACTIONAL DIFFERENTIAL EQUATIONS
}

\author{
KAI DIETHELM AND NEVILLE J. FORD
}

ABSTRACT. This note is intended to clarify some important points about the well-posedness of terminal value problems for fractional differential equations. It follows the recent publication of a paper by Cong and Tuan in this journal in which a counter-example calls into question the earlier results in a paper by this note's authors. Here, we show in the light of these new insights that a wide class of terminal value problems of fractional differential equations is wellposed and we identify those cases where the well-posedness question must be regarded as open.

1. Introduction. In the paper [6], the authors of this note discussed the important question of whether neighbouring solutions of a Volterra integral equation (possibly with a singular kernel) can intersect. A primary motivation for this work was to establish whether the terminal value problem for a fractional differential equation is wellposed. The approach stems from the observation that, for an ordinary differential equation, two solutions coincide everywhere or do not intersect anywhere. However the fractional derivative, being non-local away from the origin, opens up the possibility that neighbouring solutions may intersect and this would mean that a unique solution would not be completely determined by the solution value at some time point other than the origin. In other words, a terminal value problem might, in principle, be ill-posed.

1991 AMS Mathematics subject classification. 34A08, 45D05, 45E10, 45G05, 45G15, 45M99.

Keywords and phrases. Fractional differential equation; terminal value problem; Volterra integral equation; well-posedness; existence of solutions; uniqueness of solutions; separation of solutions.

Corresponding author: Neville J. Ford.

Received by the editors 14 March, 2017. 
The precise formulation of the problem under consideration is based on Caputo's definition of a fractional derivative,

$$
D_{*, 0+}^{\alpha} y(t)=\frac{1}{\Gamma(n-\alpha)} \int_{0}^{t}(t-s)^{n-\alpha} y^{(n)}(s) d s
$$

where $\alpha>0$ and $n=\lceil\alpha\rceil$, cf. [3, Section 3]. Classical terminal value problems for associated differential equations usually refer to the case $0<\alpha<1$ and then have the form

$$
D_{*, 0+}^{\alpha} y(t)=f(t, y(t)), \quad y(T)=y^{*}
$$

with some $T>0$ and a prescribed value $y^{*} \in \mathbb{R}$. In such a case, one is usually interested in the solution on the interval $[0, T]$; the fact that the solution's function value is prescribed at the end point of the interval of interest and not — as is traditionally done [4] — at the starting point gives rise to the denomination of such a problem as a terminal value problem. The relation of the problem (1) to the theory of Volterra integral equations is established by the well known fact [4] that a continuous function $y$ solves the differential equation that occurs in Eq. (1) if and only if there exists some $y_{0} \in \mathbb{R}$ such that

$$
y(t)=y_{0}+\frac{1}{\Gamma(\alpha)} \int_{0}^{t}(t-s)^{\alpha-1} f(s, y(s)) d s
$$

The key result of our paper [6] was contained in Theorem 3.1 of that paper which attempted to show that, under fairly weak conditions, two neighbouring solutions of a Volterra integral equation coincide either everywhere or nowhere. An application of that result appeared to give rise to the following statement regarding fractional differential equations [6, Theorem 4.1]:

Theorem 1. Let $0<\alpha<1$, and assume $f:[0, T] \times[c, d] \rightarrow \mathbb{R}$ to be continuous and satisfy a Lipschitz condition with respect to the second variable. Consider two solutions $y_{1}$ and $y_{2}$ to the differential equation

$$
D_{*, 0+}^{\alpha} y_{j}(t)=f\left(t, y_{j}(t)\right) \quad(j=1,2),
$$

subject to the initial conditions $y_{1}(0)=y_{10} \neq y_{20}=y_{2}(0)$. Then, for all $t$ where both $y_{1}(t)$ and $y_{2}(t)$ exist, we have $y_{1}(t) \neq y_{2}(t)$. 
From this result, one may conclude that a wide class of terminal value problems for fractional differential equations of the form (1) is indeed well-posed. As pointed out by Cong and Tuan [2] (see below), it is important to note here that the result is only claimed for the case of a scalar differential equation, i.e. we do not allow $y$ to be an $N$-dimensional vector with $N>1$ and $f$ to be a mapping from a suitable subset of $\mathbb{R}^{N+1}$ to $\mathbb{R}^{N}$.

Both before the publication of the paper [6] and after there have been significant numbers of outputs based on the assumption that boundary value problems are well-posed for the fractional calculus. Accordingly, the recent publication of the paper [2] whose authors point out, through a counterexample and new proof, that Theorem 3.1 of [6] (on which the fundamental result given in Theorem 1 regarding the well-posedness of the terminal value problems is based) is valid only under additional hypotheses, makes it important to assess the implications of these new insights for those working on fractional differential equations.

2. The new results. In [2], Cong and Tuan show that Theorem 3.1 of [6] is true only under additional conditions. However, the most important cases practically arise where the additional assumptions hold. So, for example they give the following result [2, Theorem 5].

Theorem 2. Let $0<\alpha<1$ and let $J=[0, T]$ for some $T>0$. Consider the equation

$$
D_{*, 0+}^{\alpha} x(t)=f(t, x(t))
$$

where $f: J \times \mathbb{R} \rightarrow \mathbb{R}$ satisfies the Lipschitz condition

$$
|f(t, x)-f(t, y)| \leq L(t)|x-y|
$$

for all $t \in J$ and all $x, y \in \mathbb{R}$ with some function $L \in C(J)$. Then for any two different initial values $x_{10}, x_{20} \in \mathbb{R}$ the trajectories of the corresponding solutions to (3) do not meet on $J$.

It thus immediately follows that Theorem 1 (i.e., Theorem 4.1 of [6]) is correct although its original proof given in [6] is erroneous. 
3. Implications for fractional terminal value problems. For single term scalar fractional terminal value problems of order $\alpha \in(0,1)$, Theorem 2 provides the theory we require for the problem to be wellposed under simple assumptions (continuity and Lipschitz continuity of $f$ ).

When it comes to equations of order $\alpha>1$, the number of initial conditions required in an initial value problem associated to the differential equation (3) is $m:=\lceil\alpha\rceil>1$. Even in the very simple case of the initial value problems

$$
D_{*, 0+}^{\alpha} y_{j}(t)=\lambda y_{j}(t), \quad y_{j}(0)=y_{j 0}, \quad y_{j}^{\prime}(0)=0 \quad(j=1,2)
$$

with $y_{10} \neq y_{20}$ and $1<\alpha<2$ it is known [6, Example 5.1] that the situation regarding the well-posedness is completely different: The solutions $y_{1}$ and $y_{2}$ intersect at least once. Thus, from the point of view of the associated terminal value problem, we may lose the existence or the uniqueness of the solution, and so the problem becomes ill-posed.

Similarly, in the vector valued case it follows from [2, Theorem 23 and Remark 25] that an intersection of the solution trajectories is possible whenever $\alpha \neq 1$. Thus, in this case the well-posedness is lost too.

The fact that these two formally different problems - i.e. the vector valued setting on the one hand and the higher order problem on the other hand - exhibit the same type of behaviour can be interpreted on the basis of the fact that there exists some link between them in the sense that problems of one type may often be rewritten in the form of the other type $[5,7,8]$.

Concerning a number of other aspects, the comments listed in [6, Section 5] are not affected by the error in the earlier part of that paper; Theorem 5 of [2] proves that they are correct:

(1) The kernel of a Caputo differential operator of order $\alpha \in(0,1)$ is one-dimensional for all $t>0$.

(2) The additional condition required in order to obtain uniqueness of the solution to a given scalar fractional differential equation of order $\alpha \in(0,1)$, where the function $f$ on the right-hand side is assumed to satisfy a Lipschitz condition with respect to the second variable, may be imposed at an arbitrary point $T \geq 0$. 
(3) It is legitimate to consider a fractional differential equation subject to a (terminal) condition given at some point $T>0$ and to ask for the behaviour of its solution in the interval $[0, T)$, i.e. at the points in time that precede the observation time $T$.

4. Conclusions and open questions. The original paper [6] discussed a number of questions related to separation properties for the solutions to a general class of Volterra equations; the corresponding results for fractional differential equations can be seen as special cases thereof. As described above, the results of [2] prove that the essential results of [6] for fractional differential equations are correct. In particular, the numerical technique developed in [6, Section 6] for solving terminal value problems is justified. However, the issue of finding a (theoretical) method to compute the exact solution of such a problem remains unsolved. It seems unclear whether, e.g., a Picard iteration technique would lead to a sequence that converges towards the exact solution under sufficiently general assumptions.

When it comes to the general class of Volterra equations discussed in [6], a number of questions remains open. For the Volterra equation of Hammerstein type

$$
y(t)=y_{0}+\int_{0}^{t} p(t, s) f(s, y(s)) d s
$$

it is claimed in [6, Theorem 3.1] under very general assumptions on the kernel $p$ and the usual Lipschitz condition with respect to the second variable on $f$ that there exists at most one $y_{0}$ such that the solution to this equation satifies $y(T)=b$ with prescribed values of $T>0$ and $b \in \mathbb{R}$. This is equivalent to a non-intersection of the solutions to the Volterra equation for different values of $y_{0}$. It is clear from the results of [2] that the proof attempted in [6] is incorrect. We conjecture that the result is correct in the scalar case under certain additional assumptions on $p$ that might be related to those used in [2, Proof of Lemma 2]. In the multidimensional case (which had not been discussed in [6]), it follows from [2, Section 6] that a corresponding result cannot hold in general; it may however hold if one restricts the attention to "sufficiently short" intervals. It would be of interest to investigate whether one can give a precise account of what "sufficiently short" means in this context and whether the admissible interval length 
depends on the dimension of the space to which $y$ maps. This question may also be related to the findings of Agarwal et al. [1], in particular to their Theorem 3.3.

\section{REFERENCES}

1. R.P. Agarwal, M. Benchohra and S. Hamani, A survey on existence results for boundary value problems of nonlinear fractional differential equations and inclusions, Acta Appl. Math. 109 (2010), 973-1033.

2. N.D. Cong and H.T. Tuan, Generation of non-local fractional dynamical systems by fractional differential equations, J. Integral Equations Appl., to appear (2017).

3. K. Diethelm, Fractional differential equations, Springer, Berlin, 2010.

4. K. Diethelm and N.J. Ford, Analysis of fractional differential equations, J. Math. Anal. Appl. 265 (2002), 229-248.

5. K. Diethelm and N.J. Ford, Multi-order fractional differential equations and their numerical solution, Appl. Math. Comput. 154 (2004), 621-640.

6. K. Diethelm and N.J. Ford, Volterra integral equations and fractional calculus: Do neighbouring solutions intersect? J. Integral Equations Appl. 24 (2012), 25-37.

7. J.T. Edwards, N.J. Ford and A.C. Simpson, The numerical solution of linear multi-term fractional differential equations: Systems of equations, J. Comput. Appl. Math. 148 (2002), 401-418.

8. N.J. Ford and A.C.Simpson, The approximate solution of fractional differential equations of order greater than 1, in Proc. 16th IMACS World Congr. Sci. Comput., Appl. Math., and Simulation, M. Deville and R. Owens, eds., Paper \#213-1, Lausanne (2000).

Institut Computational Mathematics, AG Numerik, Technische UniverSität Braunschweig, Universitätsplatz 2, 38106 Braunschweig, Germany; GNS Gesellschaft für numerische Simulation mbH, Am Gaussberg 2, 38114 Braunschweig, Germany

Email address: k.diethelm@tu-bs.de; diethelm@gns-mbh.com

University of Chester, Parkgate Road, Chester, CH1 4BJ, UK

Email address: njford@chester.ac.uk 